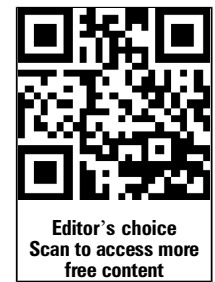

- Additional material is published online only. To view please visit the journal online (http://dx.doi.org/10.1136/ thoraxjnl-2013-203581).

${ }^{1}$ Division of Pulmonary Medicine, University Hospitals of Geneva, Geneva, Switzerland

${ }^{2}$ Division of Pulmonary Medicine, University Hospital of Lausanne, Lausanne, Switzerland

${ }^{3}$ Clinic of Thoracic Surgery, University Hospitals of Geneva, Geneva, Switzerland ${ }^{4}$ Organ Transplantation Center, University Hospital of Lausanne, Lausanne, Switzerland

${ }^{5}$ Laboratory of Virology, Division of Infectious Diseases and Division of Laboratory Medicine, University Hospitals of Geneva and Medical School, University of Geneva, Geneva, Switzerland

${ }^{6}$ Laboratory of Virology, Division of Infectious Diseases and Division of Laboratory Medicine, University Hospitals of University Hospital of Lausanne, Lausanne, Switzerland

\section{Correspondence to} Prof Laurent Kaiser,

Division of Infectious Disease, University Hospitals of Geneva, Geneva 1211, Switzerland; laurent.kaiser@hcuge.ch

Received 16 March 2013 Revised 6 August 2013 Accepted 8 August 2013 Published Online First 11 September 2013

\section{Linked}

- http://dx.doi.org/10.1136/ thoraxinl-2013-204327 - http://dx.doi.org/10.1136/ thoraxinl-2013-204610

To cite: Bridevaux P-O, Aubert J-D, Soccal PM, et al. Thorax 2014;69:32-38.

\title{
Incidence and outcomes of respiratory viral infections in lung transplant recipients: a prospective study
}

\author{
Pierre-Olivier Bridevaux, ${ }_{1}^{1}$ J-D Aubert, ${ }^{2}$ P M Soccal, ${ }^{1,3}$ J Mazza-Stalder, ${ }^{2}$ C Berutto, ${ }^{4}$ \\ T Rochat, ${ }^{1} \mathrm{~L}$ Turin, ${ }^{4} \mathrm{~S}$ Van Belle, ${ }^{5}$ L Nicod, ${ }^{2}$ P Meylan, ${ }^{6} \mathrm{G}$ Wagner, ${ }_{1}^{5}$ Laurent Kaiser ${ }^{5}$
}

\section{ABSTRACT}

Background The incidence and outcomes of respiratory viral infections in lung transplant recipients (LTR) are not well defined. The objective of this prospective study conducted from June 2008 to March 2011 was to characterise the incidence and outcomes of viral respiratory infections in LTR.

Methods Patients were seen in three contexts: studyspecific screenings covering all seasons; routine posttransplantation follow-up; and emergency visits.

Nasopharyngeal specimens were collected systematically and bronchoalveolar lavage (BAL) was performed when clinically indicated. All specimens underwent testing with a wide panel of molecular assays targeting respiratory viruses.

Results One hundred and twelve LTR had 903 encounters: 570 (63\%) were screening visits, 124 (14\%) were routine post-transplantation follow-up and 209 (23\%) were emergency visits. Respiratory viruses were identified in 174 encounters, 34 of these via BAL. The incidence of infection was 0.83 per patient-year $(95 \% \mathrm{Cl}$ 0.45 to 1.52). The viral infection rates upon screening, routine and emergency visits were $14 \%, 15 \%$ and $34 \%$, respectively $(p<0.001)$. Picornavirus was identified most frequently in nasopharyngeal (85/140; 60.7\%) and BAL specimens (20/34; 59\%). Asymptomatic viral carriage, mainly of picornaviruses, was found at $10 \%$ of screening visits. Infections were associated with transient lung function loss and high calcineurin inhibitor blood levels. The hospitalisation rate was $50 \%(95 \% \mathrm{Cl} 30 \%$ to $70.9 \%$ ) for influenza and parainfluenza and $16.9 \%$ (95\% Cl $11.2 \%$ to $23.9 \%$ ) for other viruses. Acute rejection was not associated with viral infection (OR 0.4, $95 \% \mathrm{Cl} 0.1$ to 1.3 ).

Conclusions There is a high incidence of viral infection in LTR; asymptomatic carriage is rare. Viral infections contribute significantly to this population's respiratory symptomatology. No temporal association was observed between infection and acute rejection.

\section{INTRODUCTION}

Influenza, respiratory syncytial virus (RSV), parainfluenza (PIV), human metapneumovirus (HMpV), rhinovirus, enterovirus, coronavirus, human bocavirus and adenovirus are the most frequent causes of respiratory infections in humans. These viruses circulate in the community with different seasonal patterns and can lead to serious complications, mainly in individuals with predisposing risk factors such as immunosuppression or pre-existing lung

\section{Key messages}

What is the key question?

- The incidence, risk factors and clinical outcomes of viral respiratory infections in lung transplant recipients remain poorly defined.

\section{What is the bottom line?}

- Advances in molecular viral diagnostics bring to light a high incidence of viral infections among lung transplant recipients; most of these are symptomatic and thus lead to more frequent emergency visits and hospitalisations as well as transiently decreased lung function.

\section{Why read on?}

- This large prospective study is the first to systematically examine the epidemiology, symptomatology and clinical outcomes of viral respiratory infections as diagnosed in lung-transplanted patients by means of highly sensitive molecular assays.

disease. ${ }^{1-5}$ Lung transplant recipients (LTR) are particularly prone to developing lower respiratory tract infections. This is related to their immunosuppression and also to the exposure of the graft to viral agents, impaired mucociliary function, altered lymphatic drainage and the absence of a cough reflex. These infections may have the potential to promote secondary bacterial infections, acute rejection (AR) and subsequently lead to chronic graft dysfunction.

The availability of highly sensitive diagnostic panels targeting the nucleic acids of multiple respiratory viruses now allows for the exploration of the complex relationship between specific respiratory viruses and clinical outcomes in LTR. Previous investigations and a systematic review have shown that viral infections detected by molecular assays are associated with respiratory symptoms, but their association with AR remains controversial. ${ }^{67}$ Nonetheless, both the retrospective nature of most studies and the difficulties in conducting a complete panel of molecular assays in a standardised manner allow for only weak inferences regarding the role of viruses in this patient population. The aims of our study were to investigate the 
incidence, risk factors, symptomatology and clinical outcome of respiratory viral infections detected by molecular assays in LTR and to search for an association between respiratory viral infections and biopsy-proven acute allograft rejection.

\section{METHODS}

\section{Study population and procedures}

Of 116 eligible LTR, we included 112 patients attending the Centre Universitaire Romand de Transplantation, a Swiss transplantation centre bridging two separate hospitals (University Hospitals of Geneva, Geneva and the University Hospital of Lausanne, Lausanne). All patients were followed from June 2008 to March 2011. Patient encounters comprised a scheduled post-transplant visit ('routine visit') and unscheduled visits occasioned by new symptoms or loss of lung function ('emergency visits'). As immunosuppressive medication can mask viral respiratory symptoms, we further scheduled seven 6-week screening periods covering all seasons over 2 years ('screening visit') in order to capture infections that would not be reported by LTR.

At each encounter, symptoms were recorded using studyspecific case report forms while viral screening was performed on pooled nasopharyngeal and pharyngeal swabs collected by specialised medical staff using the Centers for Disease Control and Prevention standardised procedure (http://www.cdc.gov/ pertussis/clinical/diagnostic-testing/specimen-collection.html). In addition, at each routine or emergency visit, spirometry measurements, blood tests, bronchoscopic procedures with bronchoalveolar lavage (BAL) and transbronchial biopsies (TBB) were performed according to local guidelines.

\section{Primary and secondary outcomes}

The main outcome of the study was the incidence and distribution of viral respiratory infections among LTR. Secondary outcomes included symptoms, hospitalisations, lung function changes and AR attributed to viral infections. Lung function changes between the pre-infection phase and infection were estimated based on the comparison of the forced expiratory volume in $1 \mathrm{~s}\left(\mathrm{FEV}_{1}\right)$ obtained during the 3 months prior to infection and the $\mathrm{FEV}_{1}$ measured during infection. We considered mainly biopsy-proven AR grade A2 or higher, as specific treatment is controversial for lower grades. ${ }^{8}$

\section{Viral PCR detection}

Each upper or lower respiratory specimen was screened by nucleic acid detection for the presence of influenza (A and B), viruses belonging to the Paramyxoviridae family (RSV (A and B), PIV (1-3)), HMpV, picornavirus (rhinovirus A, B and C, enterovirus), coronavirus (229E, OC43, NL63, HKU1), human bocavirus and adenovirus. At the taxonomic level, rhinoviruses and enteroviruses are two species in the Enterovirus genus and members of the human picornavirus family. As they are detected by the same assay, we use the term 'picornavirus' when describing these viruses. Screening was performed using individual two-step real-time TaqMan-based reverse transcriptase PCR (RT-PCR) or PCR assays as described previously. ${ }^{6} 9$ In brief, respiratory specimens were extracted using the HCV Amplicor Specimen Preparation kit (Roche, Rotkreuz, Switzerland) for the first year of the study and then Easymag (bioMérieux, Geneva, Switzerland), according to the manufacturers' recommendations. Eight negative controls were introduced within each of the 96-well plates and inhibition was controlled by spiking each specimen with a quantified standard using canine distemper virus. Experiments were validated only if the resulting cycling threshold (CT) value was within expected ranges. PCR detection was considered positive if duplicated by a second complete experiment on an original aliquoted specimen and with a CT value of $\leq 39$.

\section{Statistical methods}

The incidence of viral infection was estimated with Stata V11 (Statacorp, College Station, Texas, USA) using the Poisson routine. Incident rate ratios were estimated as categorical functions of the post-transplant period and bronchiolitis obliterans syndrome (BOS).

To compare predictors of viral infections, we applied generalised linear or logistic latent models using the xtreg or xtlogit commands of Stata. We chose this method to take into account the repeated measures in each LTR. ORs with CIs for AR (stage 2 or 3 ) and respiratory symptoms associated with respiratory viruses were obtained using the same approach, controlling for study site. The tacrolimus trough levels, which were abnormally distributed, were compared between virus-positive and virusnegative visits after $\log$ transformation.

Because the role of picornavirus is controversial, we carried out sensitivity analyses excluding infection with picornavirus.

\section{RESULTS}

\section{Patients and baseline characteristics}

On average, patients received lung transplants a mean (SD) of 4.0 (4.1) years before study entry; nearly one-third $(n=31$; $27.7 \%$ ) were transplanted within the previous 3 months. Conditions leading to lung transplantation were mainly chronic obstructive lung disease (42.8\%) and cystic fibrosis (27.7\%); median age at transplantation was 49.5 years. Most patients were on triple immunosuppressive therapy and 16\% had BOS stage 1 or more at study entry (table 1). Patients had a median of five study visits and an average follow-up of 827 days (range 52-1037). A total of 903 visits were analysed: 570 (63.1\%) during screening periods defined by the study protocol; 124 $(13.7 \%)$ routine post-transplantation visits; and 209 (23.2\%) due to new or worsening respiratory symptoms (see online supplementary appendix table 1).

\section{BAL and transbronchial biopsy}

BAL fluid was obtained in 276 cases. In 153 specimens (55.4\%), no pathogen was detected. A virus was present in 34 (12\%), at least one bacterium in $73(26.4 \%)$ and at least one fungus in 23 (8.3\%). Thirty-two specimens (11.6\%) were polymicrobial. Multiple infection was found in 14/34 virus-positive BAL specimens: Pseudomonas aeruginosa $(\mathrm{n}=6)$, Aspergillus spp $(\mathrm{n}=3)$, Candida spp $(\mathrm{n}=3)$, Escherichia coli $(\mathrm{n}=2)$, Streptococcus pneumoniae $(\mathrm{n}=1)$ and non-tuberculous mycobacteria $(\mathrm{n}=1)$.

Two hundred and thirty-five TBB specimens were classified according to the pathological AR grade (see online supplementary appendix tables 1 and 2). LTR with moderate or severe AR were frequently treated for rejection (A2 83.3\%; A3 94.9\%), while those with low-grade AR were rarely treated (A0 6.7\%; A1 7.9\%).

\section{Incidence of viral infections}

The viral infection rate was $19.3 \%(174 / 903)$ overall and $12.3 \%$ $(34 / 276)$ in the BAL subgroup. Sixty-eight of 112 LTR (61\%) had one or more viral infections (see online supplementary appendix figure 1). Three had 10 or 11 viral infections; all were BOS stage 3 and at least 5 years post-transplant. The incidence of viral infection affecting upper and/or lower airways was 0.83 per patient-year (95\% CI 0.45 to 1.52 ). Patients transplanted within 
Table 1 Main characteristics of lung transplant recipients at study entry $(n=112)$

\begin{tabular}{lc}
\hline Female/male & $\mathrm{n} 58 / 54$, \\
& median (range) \\
Age at lung transplantation (years) & $49.5(11-66)$ \\
Age at study entry (years) & $53.5(16-70)$ \\
& $\mathrm{n}(\%)$ \\
Diagnosis leading to lung transplantation & \\
Chronic obstructive pulmonary disease & $48(42.0)$ \\
Cystic fibrosis & $31(27.7)$ \\
Idiopathic pulmonary fibrosis & $6(5.4)$ \\
Pulmonary hypertension & $9(8.0)$ \\
Other & $18(16.1)$ \\
Time since lung transplantation & \\
$<3$ months & $31(27.7)$ \\
3 months to 1 year & $10(8.9)$ \\
1-3 years & $18(16.1)$ \\
3-5 years & $15(13.4)$ \\
$5-10$ years & $27(24.1)$ \\
$>10$ years & $11(9.8)$ \\
Immunosuppressive therapy & \\
Calcineurin inhibitors & $107(96)$ \\
Mycophenolate mofetil or azathioprine & $112(100)$ \\
mTOR inhibitors & $5(4)$ \\
Systemic corticosteroids & $110(98)$ \\
Bronchiolitis obliterans syndrome (BOS) stage & \\
BOS 0 & $71(63.4)$ \\
BOS Op & $20(17.9)$ \\
BOS 1 & $9(8.0)$ \\
BOS 2 & $3(2.7)$ \\
BOS 3 & $6(5.4)$ \\
BOS undetermined & $3(2.7)$ \\
\hline mTOR, mammalian target of rapamycin. & \\
&
\end{tabular}

the previous year did not have a higher incidence compared with those more than 1 year post-transplant (incidence rate ratio (IRR) 1.57 (95\% CI 0.63 to 3.93); $\mathrm{p}=0.329$ ). Incidence was not associated with baseline BOS stage, comparing BOS 0 with later stages (IRR 1.76 (95\% CI 0.72 to 4.30$) ; \mathrm{p}=0.215$ ).

\section{Distribution of viral infections}

Picornavirus was the most frequently detected virus for all visit types $(105 / 174,60.3 \%)$ and among positive BAL specimens (20/ $34,59 \%$ ) (figure 1). Sequence analysis and specific assays revealed that almost all picornaviruses were in fact rhinoviruses (data not shown). All viruses had a relatively similar distribution among upper and lower respiratory specimens (figure 1A). During the study period, 10 LTRs repeatedly screened positive for picornavirus. In three cases picornavirus infection lasted from 120 to 180 days, while it was shorter in others. However, we could not distinguish between re-infection and protracted infection as genome-sequencing techniques were not employed in this study.

Viruses other than picornavirus were four times more likely to be identified at emergency visits $(40 / 72,65.6 \%)$ than at routine or screening phase visits $(29 / 102,28.4 \%$; OR 3.7 (95\% CI 1.7 to 8.1 ); $\mathrm{p}=0.010$ ) (figure $1 \mathrm{~B}$ ). Adenovirus, PIV, RSV and $\mathrm{HMpV}$ were also more frequently detected at emergency visits.

At the screening visits, $48 \%$ of patients reported new or worsening symptoms (figure 2 ). The proportion of positive viral tests was $10.0 \%$ (range $2.3-20.0 \%$ ) for those without new symptoms and $19.6 \%$ (range 6.4-29.7\%) for those with new symptoms.

Strong seasonal patterns were observed-for example, viral infections were less frequent during the summer and influenza cases were observed mainly in February to March (figure 2).

\section{Factors associated with viral infections}

Higher tacrolimus serum trough levels were observed in patients with concurrent viral infections $(p=0.015$; table 2$)$. Time since transplantation and BOS stage were not associated with viral

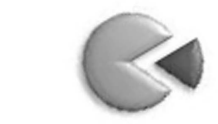

Positive, $n=159$ (18\%)

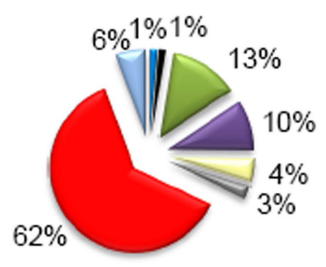

Positive, $n=34$ (12\%)

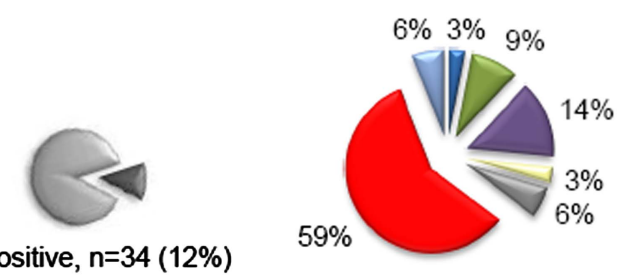

BAL specimens, $\mathbf{n}=\mathbf{2 7 6}$

Regular visits $(n=124)$

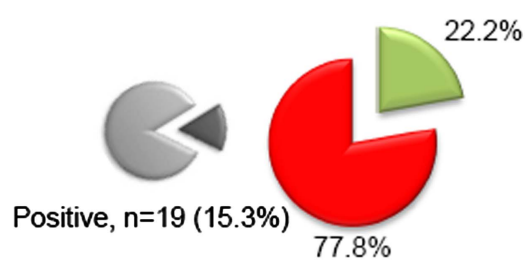

Overall proportion of $\mathbf{Q}$ positive or negative (RT)-PCR 4

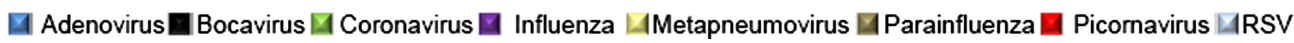

Figure 1 Distribution of viruses in lung transplant recipients. (A) Viruses recovered in upper and lower respiratory specimens. (B) Viruses recovered according to visit type. 


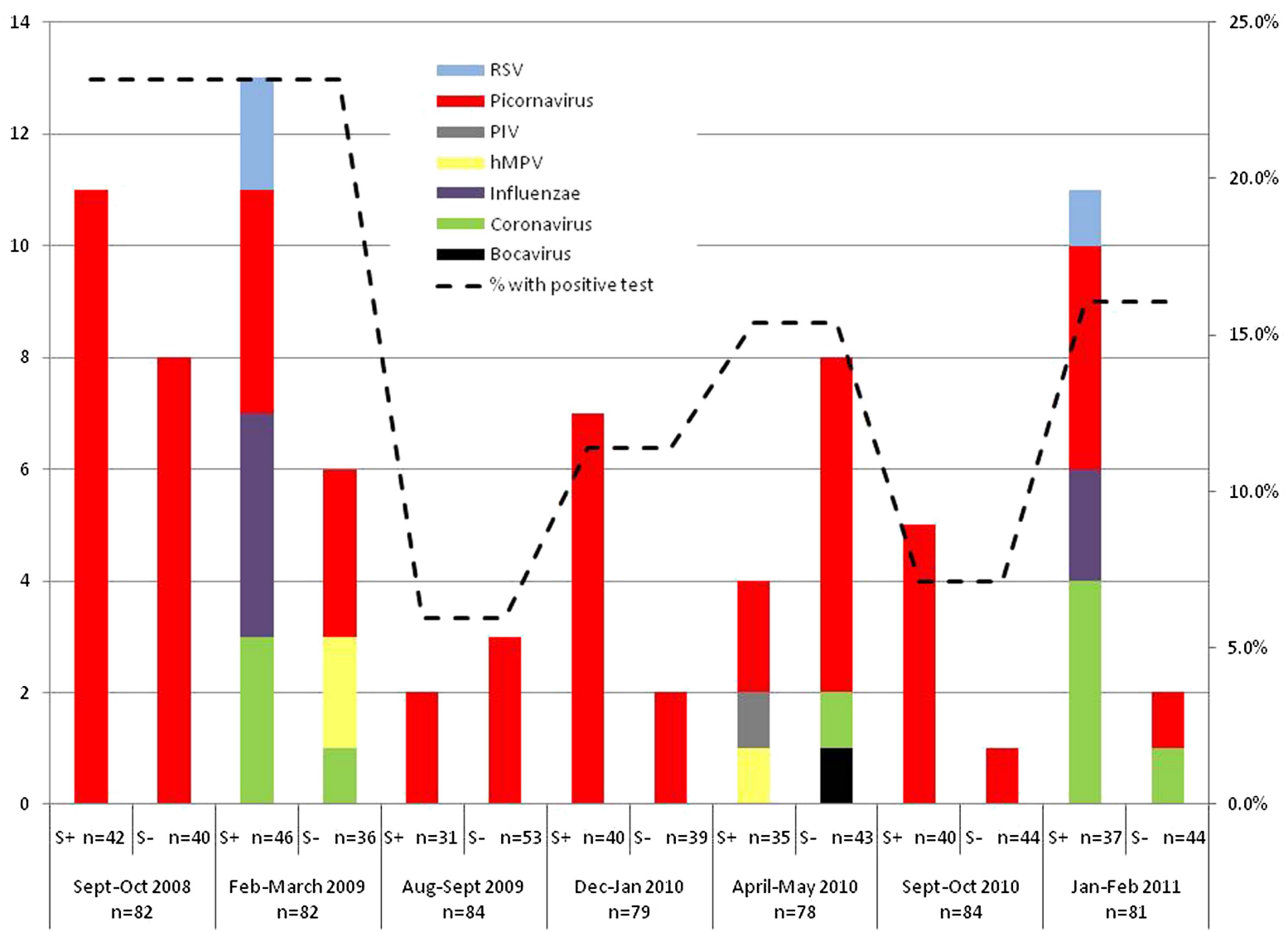

Figure 2 Overall percentage, number and type of positive viral test by screening period and presence of symptoms: y right axis: number of positive tests; y left axis: percentage of positive tests during the screening period. $S_{ \pm}$, presence or absence of new symptoms.

respiratory tract infection. Patients $\geq 1$ year post-transplant, however, were five times more likely to have lower respiratory infections detected via BAL specimens than those transplanted within the previous year.
Viral infections were more likely to be detected during an emergency visit $(34.0 \%$ vs $14.6 \%, \mathrm{p}=0.001)$ compared with a screening period and when new symptoms were reported (OR 2.9 (95\% CI 1.9 to 4.6 ), figure 3 ). Fever and sore throat were

Table 2 Factors associated with respiratory tract viral infection identified by reverse-transcription PCR in lung transplant recipients

\begin{tabular}{|c|c|c|c|c|}
\hline & \multicolumn{2}{|c|}{$\begin{array}{l}\text { Visits with upper (NPS) or lower (BAL) } \\
\text { respiratory tract viral searches }(n=903)\end{array}$} & \multicolumn{2}{|c|}{$\begin{array}{l}\text { Visits with lower (BAL) respiratory tract } \\
\text { viral searches }(n=276)^{*}\end{array}$} \\
\hline & $\begin{array}{l}\text { Negative } \\
\mathrm{N}=729\end{array}$ & $\begin{array}{l}\text { Positive } \\
\mathrm{N}=174(19.3 \%)\end{array}$ & $\begin{array}{l}\text { Negative } \\
\mathrm{N}=242\end{array}$ & $\begin{array}{l}\text { Positive } \\
\mathrm{N}=34(12.3 \%)\end{array}$ \\
\hline \multicolumn{5}{|l|}{ Time since transplantation } \\
\hline$<3$ months, $\mathrm{n}(\%)$ & 72 & $6(7.7)$ & 61 & $1(1.6)$ \\
\hline 3 months to 1 year, $n(\%)$ & 120 & $24(16.7)$ & 82 & $6(6.8)$ \\
\hline $1-3$ years, $n(\%)$ & 126 & $38(23.2)$ & 48 & $11(18.6)$ \\
\hline \multirow[t]{2}{*}{$>3$ years, $\mathrm{n}(\%)$} & 411 & $106(20.1)$ & 51 & $16(23.9)$ \\
\hline & $p=0.296 t$ & & $p=0.001 t$ & \\
\hline Tacrolimus level, $\mu \mathrm{g} / \mathrm{L}$, median (IQR) & $\mathrm{n}=397,8.7(3.2)$ & $\mathrm{n}=111,9.9(4.4)$ & $\mathrm{n}=139,8.9(3.8)$ & $\mathrm{n}=21,10.7(3.1)$ \\
\hline \multirow[t]{2}{*}{ Log-transformed tacrolimus level } & $2.18(0.36)$ & $2.27(0.32)$ & $2.21(0.36)$ & $2.32(0.28)$ \\
\hline & $p=0.015 \dagger$ & & $\mathrm{p}=0.092 \dagger$ & \\
\hline \multirow[t]{2}{*}{ Treatment for acute rejection during the preceding 3 months, $\mathrm{n}(\%)$} & 65 & 14 (17.7) & 64 & $9(12.3)$ \\
\hline & $p=0.753 \dagger$ & & $p=0.998 \dagger$ & \\
\hline \multicolumn{5}{|l|}{ Study phase } \\
\hline Screening period visits, $n$ (\%) & 487 & $83(14.6)$ & 49 & $4(7.6)$ \\
\hline Routine visits, n (\%) & 105 & $19(15.3)$ & 113 & $7(5.8)$ \\
\hline \multirow[t]{2}{*}{ Emergency visits, n (\%) } & 137 & $72(34.5)$ & 80 & $23(22.3)$ \\
\hline & $p<0.001$ & & $p<0.001$ & \\
\hline
\end{tabular}


Figure 3 OR with $95 \%$ Cls of viral respiratory infection (nasopharyngeal or bronchoalveolar lavage specimens) for specific symptoms; y axis: OR controlled for study site and repeated measures.

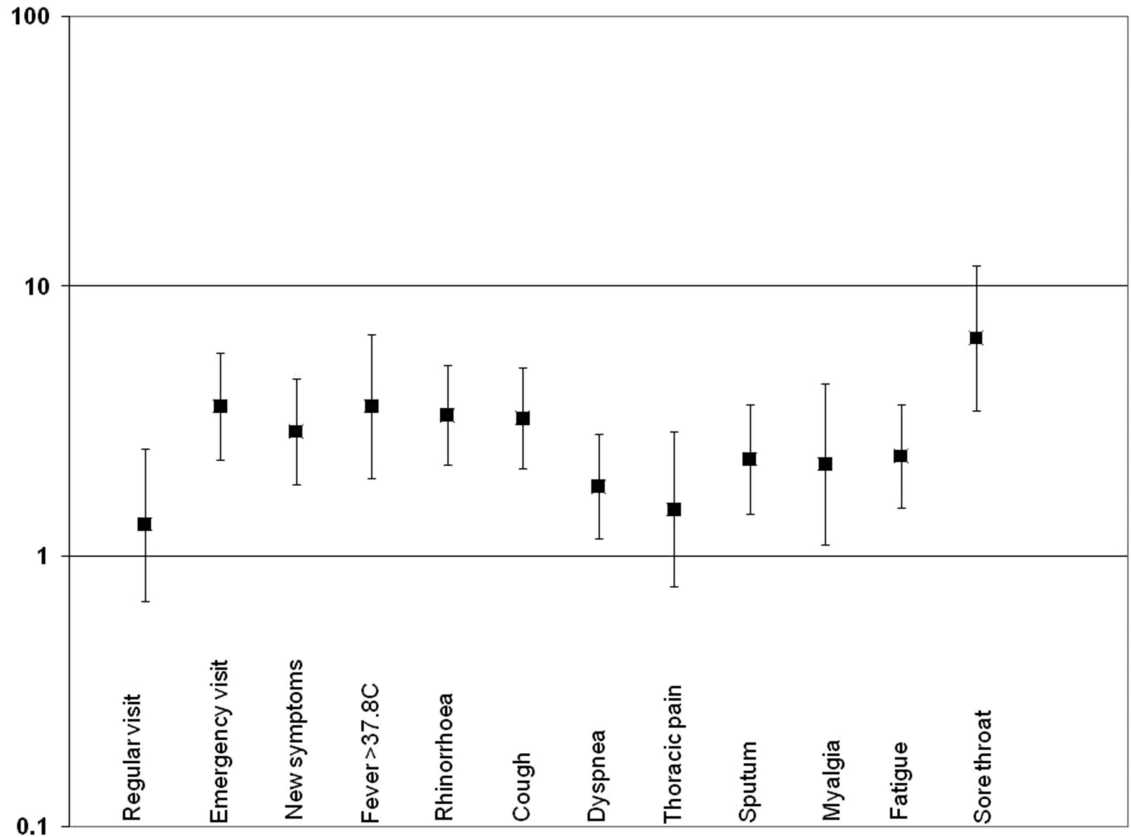

strongly associated with viral infections (43.1\% and 49.3\%, respectively); rhinorrhoea and cough were also very frequent (figure 3). Among all visits where BAL was performed, an emergency visit (OR 6.2 (95\% CI 1.5 to 26.2 )) and sputum production (OR 3.9 (95\% CI 1.4 to 10.6$)$ ) were significantly associated with viral infection. Serum C-reactive protein and procalcitonin levels did not appear to be associated with viral infection (see online supplementary appendix table 3 ).

\section{Symptoms and short-term outcomes}

Table 3 demonstrates the symptomatology, hospitalisation rate and lung function change associated with each viral agent. Of note, one-third of cases with picornavirus infection did not report any new or worsening symptoms. The most prevalent symptom for picornavirus was rhinorrhoea (43.0\%), while fever $(14.3 \%)$ and myalgia (7.6\%) were uncommon. At the other end of the spectrum, influenza, RSV and PIV were almost systematically associated with new symptoms. Fever was present in $61.1 \%$ of patients with influenza. The sum of new symptoms, ranging from 0 to 9 , varied according to specific viruses. Lower scores were found for picornavirus (mean (SD) 2.2 (2.3) new symptoms) and human bocavirus (mean (SD) 2.5 (3.5), and higher scores for influenza (4.4 (3.0), PIV (4.3 (1.6) and RSV (3.9 (2.4)). Overall, new infiltrates on chest x-ray were rare, except for patients with influenza (22.2\%). The hospitalisation rate varied markedly among viruses. Hospitalisation rates during viral respiratory infections were $50.0 \% \quad(95 \%$ CI $30.0 \%$ to $70.1 \%$ ) for transplant recipients with influenza or PIV, $18.9 \%$ (95\% CI $12.5 \%$ to $26.8 \%$ ) for those with picornavirus, coronavirus or human bocavirus, and $12.9 \%$ (95\% CI $9.8 \%$ to $15.1 \%$ ) for patients without viral or bacterial infection. LTR with influenza or paramyxoviruses (RSV, PIV) were three times more likely to be hospitalised (OR 3.1 (95\% CI 1.8 to 5.1$)$; $\mathrm{p}<0.001$ ) than those without viral or bacterial infection. Patients with picornavirus or coronavirus infection tended to be more frequently hospitalised (OR 1.7 (95\% CI 0.9 to 3.3 ); $\mathrm{p}=0.080$ ) than those without viral or bacterial infections.

Among the 12 patients who died during the study, picornavirus ( 2 patients) and adenovirus (1 patient) infections were diagnosed shortly before death.

\section{Lung function changes}

Many LTR were too ill to perform acceptable quality lung function tests at an emergency visit, but function was poorer in those with viral infection. LTR with viral infection had a mean loss of $106 \mathrm{~mL}$ (95\% CI $-157 \mathrm{~mL}$ to $-56 \mathrm{~mL}$ ) on forced expiratory volume in $1 \mathrm{~s}\left(\mathrm{FEV}_{1}\right)$ compared with pre-infection lung function. At recovery, function returned to pre-infection values. Table 3 and online supplementary appendix figure 2 show lung function changes compared with pre-infection values. When excluding picornavirus from the analyses, lung function change was within a similar range $(-106 \mathrm{~mL}(95 \% \mathrm{CI}-189 \mathrm{~mL}$ to $-23 \mathrm{~mL})$.

\section{Acute rejection (AR)}

Biopsy-proven AR was not associated with concurrent viral infection (OR 0.4 (0.1 to 1.3$)$ ), nor was initiation of AR therapy within the next 3 months (OR 0.8 (0.4 to 2.0$)$ ) (see online supplementary appendix table 4). Table 2 in the online appendix details the frequency of virus associated with acute vascular or bronchiolar rejection grades. There was still no association when picornavirus was excluded from the analysis. The individual clinical course of an initial viral infection identified via BAL is shown in online supplementary appendix figure 3. Following the incident lower respiratory tract infection, only three cases with picornavirus, influenza or coronavirus experienced AR at 90,120 and 150 days, respectively (table 3 ). One additional patient had an upper respiratory picornavirus infection 90 days before AR.

\section{DISCUSSION}

We show that respiratory viral infections are very common in LTR. Picornavirus, in particular rhinovirus, was the most frequent causative agent recovered in both upper and lower respiratory specimens, followed by coronavirus and influenza. However, the clinical course of each of these viral infections was variable. Of note, influenza and paramyxoviruses were associated with a higher symptom load, contributing to $50 \%$ of emergency visits and hospitalisations. Symptoms most often encountered with picornavirus or coronavirus are cough and rhinorrhoea, but one-third of our study population infected with these agents were asymptomatic. Conversely, influenza, paramyxovirus and adenovirus, which are less common, were 


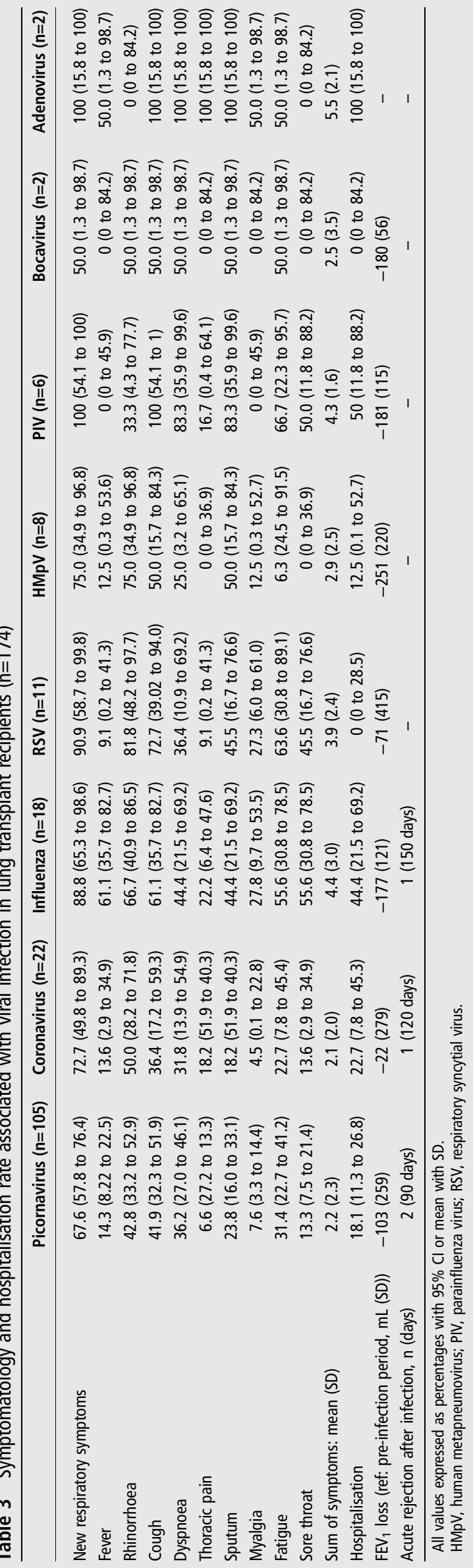

associated with a higher burden of symptoms. Almost all cases with these viruses reported upper respiratory symptoms; approximately one-half had systemic signs or symptoms such as fever or fatigue.

Interestingly, picornavirus circulates year-round with an alternating seasonal pattern. ${ }^{10}$ This contrasts with other viruses like influenza which are found during seasonal epidemics only. We further observed that the epidemiology and impact of viruses differed in the upper and lower respiratory tracts. Picornavirus and coronavirus were largely predominant in the upper respiratory tract and two-thirds were found during routine or screening visits. Again, this suggests a limited clinical impact of picornavirus and coronavirus when recovered in the upper respiratory tract, as these were not patient-initiated visits.

Picornavirus and coronavirus were also predominant in the lower respiratory tract. Of 34 viral infections detected in 276 BAL samples, 20 (59\%) were caused by picornaviruses, mainly rhinoviruses. Compared with influenza or paramyxoviruses which are known to infect the lower respiratory tract, this proportion is higher than previously reported. ${ }^{9} 1112$ This may be due in part to the sensitivity of the assays used, the population selected or the study design: all cases were systematically screened, even in the absence of overt clinical symptoms. ${ }^{13-15}$ The lower respiratory tropism of rhinovirus and its ability to promote exacerbations have been documented, particularly in patients with asthma or chronic obstructive pulmonary disease. ${ }^{16}{ }^{17}$ In LTR, rhinovirus has been shown to adapt to the lower respiratory tract and cause protracted and chronic infections. ${ }^{18} 19$ We further describe distinct symptom patterns triggered by specific respiratory viruses. Our study supports previous observations that systemic signs and symptoms such as fever, fatigue and myalgia are often reported for influenza or RSV, but rarely for picornavirus: $60 \%$ of influenza cases presented with fever compared with fewer than $15 \%$ of picornavirus cases. Nevertheless, symptom overlap in our study population limited our ability to accurately predict the virus type. Our study confirms that immunosuppressed patients manifest fever inconsistently. ${ }^{20}$ This contrasts with community-based studies of influenza where subjects are immunocompetent and not exposed to steroids and report a prevalence of fever of $68-86 \%{ }^{21}$ Lower FEV $_{1}$ or forced vital capacity (FVC) compared with pre-infection values were observed in patients with viral infection. Mallia $e t a l^{22}$ recently reported that rhinovirus infection in patients with chronic obstructive pulmonary disease resulted in decreased peak flow without significant loss in $\mathrm{FEV}_{1}$ or FVC. A more significant loss of function was observed in cystic fibrosis patients with viral infection, mostly due to rhinovirus. ${ }^{23}$ Although the inability to obtain spirometry measurements in acutely ill patients is an unavoidable limitation, our study supports previous reports showing that viral infection acutely affects function in LTR. ${ }^{24}$

It has been theorised that viral infections promote acute graft rejection. ${ }^{8}$ While some case series suggest an association between the two, ${ }^{25}{ }^{26}$ other studies-some prospective-have found no association. ${ }^{6}{ }^{27} \mathrm{~A}$ meta-analysis could not confirm this link. ${ }^{7}$ Similarly, the present prospective study shows no temporal relation between respiratory viruses and biopsy-proven AR. However, this finding does not preclude an association between viral infection and chronic graft rejection.

We identified elevated tacrolimus levels as a possible risk factor for viral infection. To our knowledge, no prospective study has yet employed both a quantitative evaluation of immunosuppression and concomitant viral screening in LTR. As calcineurin inhibitors weaken the lymphocyte-mediated immune response, it is plausible that viruses are not contained, leading to prolonged shedding and protracted infection. 
The study included prespecified periods during which transplant recipients were screened for viral infection, independent of symptomatology. Asymptomatic carriage of viral RNA or DNA in the upper respiratory tract was found at $10 \%$ of screening visits, most belonging to picornavirus. While viral RNA, mostly from rhinovirus, was detected in up to $30 \%$ of asymptomatic immunocompetent children, ${ }^{10} 2829$ Johnston et al ${ }^{30}$ found only $4 \%$ of asymptomatic adults to be carrying rhinoviruses. However, the true prevalence of asymptomatic viral carriage in immunocompetent adults remains uncertain and thus cannot be compared with the rate observed in our study population during screening periods.

The strengths of our study are its prospective design with a full record of all visits over more than 30 months, as well as the use of an extensive panel of RT-PCR viral tests. It is unlikely that clinically significant viral infections were missed in our cohort. The seven screening periods covering all four seasons offered a unique opportunity to evaluate the incidence of asymptomatic viral infection. The fact that lung function could not be evaluated in many acutely ill patients is a limitation. This potential bias may lead to an underestimation of lung function loss due to acute viral infection, but does not alter our conclusions.

This large prospective study of LTR found that respiratory viral infections could be diagnosed in one-third of emergency visits. Viral infections transiently diminish lung function and lead to more hospitalisations, often including invasive procedures such as bronchoscopy, but are not associated with AR. Asymptomatic viral infection is rare and occurs most often with picornavirus. Specific antiviral therapy in LTR is limited and its efficacy as yet unproven. ${ }^{3} 313226$ The incidence and clinical impact of viral infections in LTR demonstrated by this study suggest a need for trials investigating both preventive and protective interventions in this population.

Acknowledgements The authors are indebted to the physicians and collaborators at the Centre Universitaire Romand de Transplantation in Lausanne and Geneva for their active participation in the study: Florian Charbonnier, Jérome Plojoux, Isabelle Frésard, Grégoire Gex, Jacques Pralong, Igor Widdiker, Leslie Noirez, Antoine Pasche, Marie Hofer, Elise Dupuis-Lozeron (statistician) and Alban Lovis.

Contributors $\mathrm{P}-\mathrm{OB}$ designed the study, analysed the data and drafted the manuscript. J-DA designed the study, supervised patient enrolment and drafted the manuscript. PMS designed the study and supervised patient enrolment. JM-S and CB enrolled patients. TR and LN participated in study organisation. LT and SVB performed the laboratory analyses. PM participated in specimen collection. GW collected patient data, supervised the database and analysed the data. LK designed, planned and supervised the study, obtained the funding, analysed the data and drafted the manuscript. All authors have contributed to the manuscript and approved the final version.

Funding The study was supported by the Swiss National Science Foundation (grant 32003B_127160 to LK), the Clinical Research Center, University Hospitals of Geneva and Faculty of Medicine, Geneva and the Louis-Jeantet Foundation. The funding sources had no role in the study design, data collection and analysis, decision to publish or preparation of the manuscript.

Competing interests None.

Ethics approval Ethics approval was obtained from the Geneva University Hospitals Ethics Committee and the University Hospital of Lausanne Ethics Committee.

Patient consent Obtained.

Provenance and peer review Not commissioned; externally peer reviewed.

\section{REFERENCES}

1 Nichols WG, Peck Campbell AJ, Boeckh M. Respiratory viruses other than influenza virus: impact and therapeutic advances. Clin Microbiol Rev 2008;21:274-90.

2 Pavia AT. Viral infections of the lower respiratory tract: old viruses, new viruses, and the role of diagnosis. Clin Infect Dis 2011:52(Suppl 4):S284-89.

3 Shah JN, Chemaly RF. Management of RSV infections in adult recipients of hematopoietic stem cell transplantation. Blood 2010:117:2755-63.
4 Ison MG. Antiviral therapies for respiratory viral infections in lung transplant patients. Antivir Ther 2012;17(1 Pt B):193-200.

5 Campbell AP, Chien JW, Kuypers J, et al. Respiratory virus pneumonia after hematopoietic cell transplantation (HCT): associations between viral load in bronchoalveolar lavage samples, viral RNA detection in serum samples, and clinical outcomes of HCT. J Infect Dis 2010;201:1404-13.

6 Soccal PM, Aubert JD, Bridevaux PO, et al. Upper and lower respiratory tract viral infections and acute graft rejection in lung transplant recipients. Clin Infect Dis 2010;51:163-70.

7 Vu DL, Bridevaux PO, Aubert JD, et al. Respiratory viruses in lung transplant recipients: a critical review and pooled analysis of clinical studies. Am J Transplant 2011:11:1071-8.

8 Stewart S, Fishbein MC, Snell Gl, et al. Revision of the 1996 working formulation for the standardization of nomenclature in the diagnosis of lung rejection. $J$ Heart Lung Transplant 2007;26:1229-42.

9 Garbino J, Soccal PM, Aubert JD, et al. Respiratory viruses in bronchoalveolar lavage: a hospital-based cohort study in adults. Thorax 2009;64:399-404.

10 Lee WM, Lemanske RF Jr, Evans MD, et al. Human rhinovirus species and season of infection determine illness severity. Am J Respir Crit Care Med 2012;186: 886-91.

11 Garbino J, Inoubli S, Mossdorf E, et al. Respiratory viruses in HIV-infected patients with suspected respiratory opportunistic infection. AIDS 2008;22:701-5.

12 Kherad O, Kaiser L, Bridevaux PO, et al. Upper-respiratory viral infection biomarkers, and COPD exacerbations. Chest 2010;138:896-904.

13 Jennings $L C$, Anderson TP, Beynon KA, et al. Incidence and characteristics of viral community-acquired pneumonia in adults. Thorax 2008;63:42-8.

14 Choi SH, Hong SB, Ko GB, et al Viral infection in patients with severe pneumonia requiring intensive care unit admission. Am J Respir Crit Care Med 2012:186:325-32.

15 Templeton KE, Scheltinga SA, van den Eeden WC, et al. Improved diagnosis of the etiology of community-acquired pneumonia with real-time polymerase chain reaction. Clin Infect Dis 2005:41:345-51.

16 Wark PA, Johnston SL, Bucchieri F, et al. Asthmatic bronchial epithelial cells have a deficient innate immune response to infection with rhinovirus. J Exp Med 2005;201:937-47

17 Malmstrom K, Pitkaranta A, Carpen O, et al. Human rhinovirus in bronchial epithelium of infants with recurrent respiratory symptoms. J Allergy Clin Immunol 2006;118:591-6.

18 Kaiser L, Aubert JD, Pache JC, et al. Chronic rhinoviral infection in lung transplant recipients. Am J Respir Crit Care Med 2006;174:1392-9.

19 Tapparel C, Cordey S, Junier T, et al. Rhinovirus genome variation during chronic upper and lower respiratory tract infections. PLoS One 2011;6:e21163.

20 van den Dool C, Hak E, Wallinga J, et al. Symptoms of influenza virus infection in hospitalized patients. Infect Control Hosp Epidemiol 2008;29:314-19.

21 Call SA, Vollenweider MA, Hornung CA, et al. Does this patient have influenza? JAMA 2005:293:987-97.

22 Mallia P, Message SD, Gielen V, et al. Experimental rhinovirus infection as a human model of chronic obstructive pulmonary disease exacerbation. Am J Respir Crit Care Med 2010;183:734-42.

23 Smyth AR, Smyth RL, Tong $C Y$, et al. Effect of respiratory virus infections including rhinovirus on clinical status in cystic fibrosis. Arch Dis Child 1995;73:117-20.

24 Garbino J, Gerbase MW, Wunderli W, et al. Respiratory viruses and severe lower respiratory tract complications in hospitalized patients. Chest 2004;125 1033-9.

25 Kumar $\mathrm{D}$, Husain $\mathrm{S}$, Chen $\mathrm{MH}$, et al. A prospective molecular surveillance study evaluating the clinical impact of community-acquired respiratory viruses in lung transplant recipients. Transplantation 2010;89:1028-33.

26 Larcher C, Geltner C, Fischer H, et al. Human metapneumovirus infection in lung transplant recipients: clinical presentation and epidemiology. J Heart Lung Transplant 2005;24:1891-901.

27 Khalifah AP, Hachem RR, Chakinala MM, et al. Respiratory viral infections are a distinct risk for bronchiolitis obliterans syndrome and death. Am J Respir Crit Care Med 2004;170:181-7

28 Jansen RR, Wieringa J, Koekkoek SM, et al. Frequent detection of respiratory viruses without symptoms: toward defining clinically relevant cutoff values. J Clin Microbiol 2011:49:2631-6.

29 Garcia-Garcia ML, Calvo C, Pozo F, et al. Human bocavirus detection in nasopharyngeal aspirates of children without clinical symptoms of respiratory infection. Pediatr Infect Dis J 2008;27:358-60.

30 Johnston SL, Sanderson G, Pattemore PK, et al. Use of polymerase chain reaction for diagnosis of picornavirus infection in subjects with and without respiratory symptoms. J Clin Microbiol 1993;31:111-17.

31 Shah PD, McDyer JF. Viral infections in lung transplant recipients. Semin Respir Crit Care Med 2010;31:243-54.

32 Ison MG, Sharma A, Shepard J-AO, et al. Outcome of influenza infection managed with oseltamivir in lung transplant recipients. J Heart Lung Transplant 2008:27:282-8 